\title{
Resposta à carta ao editor referente ao artigo "Artrodese lombar intersomática anterior multinível combinada com estabilização posterior em discopatia lombar - Análise clínico-funcional prospetiva"
}

\section{Reply to Letter to Editor Regarding "Multilevel Anterior Lumbar Interbody Fusion Combined with Posterior Stabilization in Lumbar Disc Disease - Prospective Analysis of Clinical and Functional Outcomes"}

\author{
Diogo Lino Moura ${ }^{1,2}$ David Lawrence ${ }^{3}$ Josue Pereira Gabriel ${ }^{3}$
}

1 Serviço de Ortopedia, Centro Hospitalar e Universitário de Coimbra, Coimbra, Portugal

2 Fellow do Spine Institute of Ohio, Grant Medical Center, Columbus, Ohio, Estados Unidos

${ }^{3}$ Spine Institute of Ohio, Grant Medical Center, Columbus, Ohio, Estados Unidos

Rev Bras Ortop 2020;55(5):654-656.

Antes de mais nada, gostaria de agradecer pela carta ao editor relativa ao estudo prospectivo "Multilevel Anterior Lumbar Interbody Fusion Combined with Posterior Stabilization in Lumbar Disc Disease - Prospective Analysis of Clinical and Functional Outcomes."1

Um dos pontos apresentados na carta, em que são sobretudo citados artigos relativos a escolioses e não a discoartropatias degenerativas, são o risco de manipulação da coluna poder estar associados a complicações. ${ }^{2,3}$ Concordamos e está claramente documentado que a manipulação da coluna, sobretudo na correção de deformidades com curvaturas muito acentuadas, pode estar associada à perda de potenciais no intraoperatório e a risco de lesão medular. No entanto, o nosso trabalho refere-se a discoartropatias degenerativas sem escolioses relevantes, não se verificando manipulação relevante da coluna, sem ser a elevação dos espaços discais ao aplicar as caixas intersomáticas lordóticas. ${ }^{1}$

Face aos resultados clínicos e imagiológicos obtidos na nossa amostra de artrodese intersomática anterior lombar uni e multinível e à mínima incidência de complicações obtida, verificamos que, mesmo na presença significativa de fatores de risco de não união e cirurgias prévias da coluna lombar, esta

recebido

27 de Outubro de 2019

aceito

DOI https://doi.org/

$10.1055 / \mathrm{s}-0040-1701284$.

30 de Outubro de 2019
Endereço para correspondência Diogo Lino Moura, MD, Rua Fonseca Pinto, Coimbra, 3000-075, Portugal (e-mail: dflmoura@gmail.com).

opção garante excelentes resultados clínicos, funcionais e radiográficos na discoartropatia degenerativa. ${ }^{1}$

Tal como referido no artigo original, em termos de morbidade, ao contrário das vias posteriores, que implicam disseção extensa dos músculos paravertebrais, e da via lateral, que implica atravessar o psoas, a via anterior da coluna lombar não interfere com qualquer músculo da coluna vertebral e não inclui desinserções musculares. Assim, é uma via em teoria menos sangrativa, o que pode permitir uma recuperação mais rápida em termos álgicos (com menor necessidade de analgésicos) e funcionais no pós-operatório (com menor tempo de internamento) e uma coluna vertebral precocemente mais estável por não interferir com a musculatura estabilizadora. Além disso, a via anterior também não implica remoção de elementos posteriores da coluna vertebral, nem entrada no canal medular ou manipulação de raízes raquidianas para acesso ao espaço discal, diminuindo assim o risco de lesão iatrogênica e de complicações nessas importantes estruturas em comparação com as vias posteriores. ${ }^{4-8}$

Consideramos ainda que, se respeitarmos a técnica cirúrgica de artrodese intersomática anterior lombar descrita no artigo original e se a mesma for efetuada por um cirurgião

Copyright $\odot 2020$ by Sociedade Brasileira License terms de Ortopedia e Traumatologia. Published by Thieme Revinter Publicações Ltda, Rio de Janeiro, Brazil 
com formação e experiência na mesma, as complicações conseguem ser quase anuladas, podendo até ter menores complicações relativamente às habituais vias posteriores. Estes dados são confirmados por vários trabalhos relativos a esta técnica citados também no artigo original. ${ }^{1,4,5,7,9,10}$ Consideramos sobretudo, tal como o artigo originalmente escrito indica, que as vantagens biomecânicas e excelentes resultados clínico-funcionais e radiográficos desta técnica justificam a sua utilização mais frequente por cirurgiões da coluna, não devendo o risco de algumas raras complicações ser motivo para a evitarmos. Reforçamos que, tal como em qualquer outra técnica cirúrgica, a mesma deve ser naturalmente efetuada por cirurgiões com formação e experiência na abordagem de modo a se obterem os melhores resultados e minimizar as complicações. ${ }^{11-14}$

Tal como indicado no artigo original, esta técnica permite evitar manipulação direta do canal ou das raízes, baseandose sobretudo na descompressão indireta, sendo a estabilidade bastante sólida à custa das caixas intersomáticas com parafusos integrados, reforçadas posteriormente por uma fixação pedicular percutânea, tal como aliás é confirmado no trabalho citado na carta ao editor de Yeager et al. ${ }^{1,15} \mathrm{Em}$ suma, face aos nossos resultados e sua confirmação por vários outros estudos, incluindo a questão da via de abordagem e eventuais complicações, estamos convictos e mantemos a conclusão final em relação à técnica descrita. ${ }^{1}$

Conflito de Interesses

Os autores declaram não haver conflito de interesses.

\section{Referências}

1 Moura DL, Lawrence D, Gabriel JP. Multilevel Anterior Lumbar Interbody Fusion Combined with Posterior Stabilization in Lumbar Disc Disease-Prospective Analysis of Clinical and Functional Outcomes. Rev Bras Ortop (Sao Paulo) 2019;54(02):140-148

2 - Grabala P, Latalski M. Rare abdominal complications after undergoing posterior spinal fusion for progressed idiopathic scoliosis - case series and a literature review. Case Study Case Rep 2019;9(03):30-41

3 Elsamadicy AA, Koo AB, Kundishora AJ, et al. Impact of patient and hospital-level risk factors on extended length of stay following spinal fusion for adolescent idiopathic scoliosis. J Neurosurg Pediatr 2019:1-7

4 Burkus JK, Gornet MF, Schuler TC, Kleeman TJ, Zdeblick TA. Sixyear outcomes of anterior lumbar interbody arthrodesis with use of interbody fusion cages and recombinant human bone morphogenetic protein-2. J Bone Joint Surg Am 2009;91(05): 1181-1189

5 Truumees E, Majid K, Brkaric M. Anterior lumbar interbody fusion in the treatment of mechanical low back pain. Semin Spine Surg 2008;20(02):113-125

6 Mummaneni PV, Haid RW, Rodts GE. Lumbar interbody fusion: state-of-the-art technical advances. Invited submission from the Joint Section Meeting on Disorders of the Spine and Peripheral Nerves, March 2004. J Neurosurg Spine 2004;1(01):24-30

7 Strube P, Hoff E, Hartwig T, Perka CF, Gross C, Putzier M. Stand-alone anterior versus anteroposterior lumbar interbody single-level fusion after a mean follow-up of 41 months. J Spinal Disord Tech 2012;25(07):362-369

8 Zhang JD, Poffyn B, Sys G, Uyttendaele D. Are stand-alone cages sufficient for anterior lumbar interbody fusion? Orthop Surg 2012;4(01):11-14

9 Rao PJ, Loganathan A, Yeung V, Mobbs RJ. Outcomes of anterior lumbar interbody fusion surgery based on indication: a prospective study. Neurosurgery 2015;76(01):7-23, discussion 23-24

10 Sasso RC, Kitchel SH, Dawson EG. A prospective, randomized controlled clinical trial of anterior lumbar interbody fusion using a titanium cylindrical threaded fusion device. Spine 2004;29(02): 113-122, discussion 121-122

11 Chiriano J, Abou-Zamzam AM Jr, Urayeneza O, Zhang WW, Cheng $W$. The role of the vascular surgeon in anterior retroperitoneal spine exposure: preservation of open surgical training. J Vasc Surg 2009;50(01):148-151

12 Mobbs RJ, Phan K, Daly D, Rao PJ, Lennox A. Approach-related complications of anterior lumbar interbody fusion: results of a combined spine and vascular surgical team. Global Spine J 2016;6 (02):147-154

13 Wood KB, Devine J, Fischer D, Dettori JR, Janssen M. Vascular injury in elective anterior lumbosacral surgery. Spine 2010;35(9, Suppl)S66-S75

14 Quraishi NA, Konig M, Booker SJ, et al. Access related complications in anterior lumbar surgery performed by spinal surgeons. Eur Spine J 2013;22(Suppl 1):S16-S20

15 Yeager MS, Dupre DA, Cook DJ, Oh MY, Altman DT, Cheng BC. Anterior lumbar interbody fusion with integrated fixation and adjunctive posterior stabilization: A comparative biomechanical analysis. Clin Biomech (Bristol, Avon) 2015;30(08):769-774 
